\title{
How effective is augmentation with psychotherapy as a next-step option for treatment-resistant depression? ${ }^{\dagger}$
}

\section{Daniel Silman (1)}

\begin{abstract}
SUMMARY
Determining the optimum next-step treatment for the numerous patients with depression who do not adequately respond to an initial trial of medication remains a source of uncertainty in clinical practice. Although a number of psychological treatments are known to be effective for depression, their relative merits in the treatment-resistant group have not been ascertained. The Cochrane Collaboration has recently published a meta-analysis of the evidence available for the use of various psychotherapies as an adjunct to antidepressants compared with antidepressants alone in treatment-resistant depression. This article provides a commentary and appraisal of the clinical utility of these findings.
\end{abstract}

\section{KEYWORDS}

Depression; psychotherapy; cognitive behavioural therapies; statistical methodology.

Evidence suggests that the majority of depressed patients fail to adequately achieve remission after an initial trial of antidepressants (Trivedi 2006; Thase 2007). Together with issues of medication acceptability, the challenges in achieving and sustaining recovery from depressive illness present a major burden to patients, at great societal cost (McCrone 2008).

This commentary addresses the ongoing need to optimise selection of the 'next-step' options following initial antidepressant failure - referred to here as treatment-resistant depression (TRD). Current American Psychiatric Association (2010), National Institute for Health and Care Excellence (NICE) (2009) and British Association for Psychopharmacology (Cleare 2015) guidelines suggest that clinicians may consider any selection out of increasing the dose of the initial medication, switching to an alternative antidepressant (within the same or from a different class) or augmenting with a drug (such as an antipsychotic or lithium) or psychological intervention.

Psychological therapies can work by varied mechanisms in depression (explained below) and it is hoped that their benefits and those of medication may be optimised when received together. Although certain therapies have been shown to be effective for depression more broadly (e.g. cognitive-behavioural therapy (CBT) as a first-line treatment (Cuijpers 2011)), prior to this Cochrane Review (Ijaz 2018) there had been no rigorous analysis collating recent trial data for psychotherapies in treatmentresistant depression, for which there has been greater focus on pharmacological strategies (Trivedi 2006; Papakostas 2008; Shelton 2008). Previous systematic reviews in this area have not yielded clear recommendations for practice. This has been due to lack of a stringently defined clinical condition (McPherson 2005; Trivedi 2011), inclusion of uncontrolled or inappropriate interventions (McPherson 2005) or failure to find sufficient studies for meta-analysis (Stimpson 2002; Cooper 2011; McIntyre 2014). Given also the suggested preference for psychological therapies over medication in certain patient groups (McHugh 2013), this is a pressing research question.

\section{This month's Cochrane Review}

\section{Summary}

The review by Ijaz et al (2018) in this month's Cochrane Corner includes six randomised controlled trials (RCTs) involving 698 participants with treatment-resistant depression. The results showed that participants receiving augmentation of their index antidepressant treatment with psychotherapy had favourable outcomes for depressive symptoms, and for response and remission rates, compared with continued drug treatment alone, and no indication of poor acceptability. The strongest evidence was over short-term follow-up and further research is needed to guide clinical practice.

\section{An overview of psychotherapy in treatment- resistant depression}

The review aimed to determine the effectiveness of psychological therapy as part of next-step treatment strategies in adults (18-74 years) with treatmentresistant depression through meta-analysis of
Daniel Silman is a core psychiatry trainee working with Oxford Health NHS Foundation Trust in the Thames Valley region, based at Warneford Hospital, Headington, UK. He has an interest in research in the area of psychotherapy.

Correspondence Dr Daniel Silman. Email: daniel.silman@gmail.com

First received 27 May 2020

Final revision 3 Jul 2020 Accepted 7 Jul 2020

Copyright and usage (c) The Authors 2020

${ }^{\dagger}$ Commentary on... Psychological therapies for treatment-resistant depression in adults (Cochrane Corner). See this issue. 
relevant randomised controlled trials (RCTs) that specifically tested this patient group.

There is surprisingly limited consensus in defining treatment resistance in depression.

This review applied a consistent definition for included trials of non-response to at least 4 weeks' treatment with a therapeutic dose of antidepressant medication - in line with the World Psychiatric Association's earliest definition of treatment-resistant depression (non-response to $150 \mathrm{mg} /$ day of imipramine or an equivalent drug). This may be unfamiliar to some clinicians more acquainted with other classification systems for treatment-resistant depression that consider non-response to multiple courses of treatment (Fava 2003; Fekadu 2009). The extent to which someone is treatment-resistant can more accurately be assessed using a multidimensional staging model, i.e. regarding treatment resistance as a non-dichotomous entity (Ruhé 2012). Failure to respond due to medication intolerance is not considered treatment resistance and this review was stringent in excluding studies not making this distinction.

As well as being the remit of psychiatrists, treatment-resistant depression is also frequently managed within primary care (Thomas 2013) and this review included studies from both settings. The review was restricted to examining psychotherapy in those with unipolar depression, with bipolar illness and other major psychiatric comorbidities excluded.

Psychological treatments for depression may be first line in acute treatment (particularly for mild to moderate episodes) but major guidelines also recommend them as an augmentation strategy (Cleare 2015). This review also sought to determine the evidence-base for switching from medication to psychotherapy in treatment-resistant depression, which, in addition to not being in line with current guidance, could also be considered improbable in clinical practice after single treatment failure - switching is more feasibly associated with initial treatment intolerance. Perhaps not surprisingly, no RCTs could be identified comparing continued antidepressant treatment with switching to psychotherapy alone.

As succinctly summarised by the review authors, psychological treatments for depression may be categorised according to their proposed mechanism:

- psychodynamic/psychoanalytic, focusing on unconscious processes to improve understanding of the effects of past experiences on current thoughts and behaviours

- cognitive-behavioural, targeting unhelpful negative thoughts and maladaptive patterns of behaviour

- humanistic, focused on enhancing self-awareness
- integrated therapies (combining components of different models), which include interpersonal therapy (IPT) and cognitive-analytic therapy (CAT).

Evaluating psychotherapies in clinical trials will ideally take into account participant engagement and fidelity to the model, where appropriate, to maximise the validity of the proposed findings (Alvarez-Jimenez 2008). These factors were recorded in the review where possible.

\section{Method}

The search strategy principally reviewed the Cochrane Common Mental Disorders Group (CCMD) Clinical Trials Register, which is collated from routine searches of multiple databases (MEDLINE, Embase, PsycINFO, Central) and the authors also checked international trials registries for ongoing or unpublished studies. There were no date or language restrictions. A total of 4705 records were returned from the initial search, with a further 4 studies found through complementary searches of references and study author contacts. After initial screening of abstracts to remove obviously irrelevant papers, 102 full papers were reviewed for inclusion, with 20 articles pertaining to 6 studies ultimately included (Wiles 2008, 2016; Feldman 2009; Souza 2016; Nakagawa 2017; Town 2017).

Study selection was appropriately reported using a PRISMA flow diagram with characteristics of excluded studies also listed, including some wellknown studies. STAR*D (Thase 2007), for example, was excluded for progressing participants to level 2 treatments if initial citalopram treatment had been poorly tolerated - comprising some 56\% of those enrolled in medication switch options - in addition to those lacking adequate clinical response. Somewhat spuriously though, STAR*D was also flagged for not reapplying diagnostic criteria before randomisation to a next-step treatment despite participants clearly meeting clinical case definition. Overall, however, the study selection matched appropriately with the defined clinical question.

In keeping with best practice recommended by the Cochrane Collaboration, full-text article screening, data extraction from included studies and application of the 'risk of bias' tool (Higgins 2011, 2019) was independently assessed by two authors, consulting a third author to resolve discrepancies and contacting study investigators where necessary.

The primary outcome for clinical effectiveness was defined as changes in symptom severity on both selfreported (Beck Depression Inventory, BDI; Patient Health Questionnaire-9, PHQ-9) and clinicianrated (Hamilton Rating Scale for Depression, 
Pooling data from multiple clinical trials within a meta-anaIysis may give a more robust estimate of the treatment effect than any individual study. However, the key appraisal is then to assess confidence or certainty that the resulting estimate actually reflects the true effect of treatment. The factors to consider here are broadly acknowledged to determine the 'quality' of the evidence. With many potential contributors to such a judgement, the framework introduced by the Grading of Recommendations Assessment, Development and Evaluation (GRADE) Working Group has been widely adopted as a systematic and reproducible approach (Guyatt 2008).
Within this framework, clinical outcomes assessed in randomised controlled trials (RCTs) begin as high-quality evidence but then may be downgraded (to moderate, low or very low quality) on the basis of limitations in quality as assessed in five domains: risk of bias, imprecision, inconsistency, indirectness and publication bias. Determining the extent to which any of these may compromise the results is, of course, subjective and requires a degree of expert intuition, including for the clinical context. The GRADE framework also allows for identified weaknesses to be mitigated by positive indicators such as the magnitude of effect and evidence of a dose-response gradient, which themselves increase certainty.
HRSD (called HAMD in the review); MontgomeryÅsberg Depression Rating Scale, MADRS) depression scales over the duration of follow-up, most commonly reported at 6 months following treatment, although longer-term follow-up was reported in some included studies. Effect size was determined by calculating mean differences (m.d.) or standardised mean differences (s.m.d, where different measures were used for the same outcome, e.g. all selfreported measures), with 95\% confidence intervals (CIs). For the secondary dichotomous clinical outcomes, the review authors indicated that they would accept the original studies' definitions, but these are generally standardised as: response, quantified as $>50 \%$ reduction in depression scores; remission, absolute scores of $\leq 7$ on the HRSD or $\leq 10$ on the BDI. These were reported as risk ratios (RR) and number needed to treat for an additional benefit (NNTB), also with 95\% CI.

The all-cause drop-out rate was the primary indicator of acceptability, with reasons summarised where possible. Disappointingly, this review did not identify at baseline certain clinically relevant participant characteristics, in particular educational attainment and family history of mood disorder, which may influence acceptability of certain psychotherapies (Wisniewski 2007). Other secondary outcomes in the review included data on social functioning, quality of life (QoL), economic outcomes and specific adverse events when reported in the original study.

Given that the meta-analysis pooled results from different modalities of psychotherapy (i.e. the intervention arm was not uniform between studies), it was appropriate that the review authors used a random-effects model for all analyses, as this accounts for between-study variation in estimating the true effect. Heterogeneity of included studies was formally assessed with the chi $^{2}$ test and the $I^{2}$ statistic, and the GRADE approach was used to denote evidence quality for each finding (Box 1).

\section{Results}

Of the six studies meeting all inclusion criteria, all were parallel-group randomised trials studying a psychological treatment as an adjunct to treatment as usual (TAU) versus TAU alone. Three studies evaluated individual CBT, one study assessed group dialectical behaviour therapy (DBT, incorporating change-oriented cognitive-behavioural strategies) and one assessed IPT and intensive short-term dynamic psychotherapy (ISTDP). Participants continued antidepressant treatment as part of TAU but further conditions, such as changes in management and contact with professionals, may inevitably vary according to the setting and were not strictly defined. For example, the majority $(469 / 698)$ of participants included in this review came from the UK multicentre CoBalT study (Wiles 2016), where TAU was overseen by the patients' general practitioner, who could amend management, including referral to secondary care, in line with standard guidelines. Secondary care settings and other countries (USA, Canada, Japan and Brazil) were represented in the other studies.

At the key 6-month follow-up time point, when all studies collected self-reported depressive symptoms (either BDI or PHQ-9 scores, $n=635$ ), the pooled mean difference favoured the addition of psychotherapy to usual care when combining data from these scales (s.m.d. $=-0.40,95 \%$ CI -0.65 to -0.14 ,) and considering the more commonly applied BDI alone (5 studies, m.d. $=-4.07$, CI -7.01 to -1.07$)$. These findings were moderatequality evidence with little heterogeneity: $I^{2}$ of 
37 and 27\% respectively. A slightly lower but still significant reduction (m.d. $=-3.28,95 \%$ CI -5.71 to $-0.85, I^{2}=30 \%$ ) was seen in the observer-rated HRSD, but this was deemed lower-quality evidence as it aggregated data from four of the smaller studies (and not the large CBT trial) and covered all three therapy subtypes.

All six studies reported on remission rates, which showed an almost two-fold higher likelihood $(\mathrm{RR}=1.92,95 \% \mathrm{CI} 1.46-2.52)$ in the intervention arms over the short term (6 months), with a reported NNTB of 6.5. The response rate was similar but collected in only four studies and therefore deemed a lower-quality indication of favourable outcome with adjunctive psychotherapy. One study reported response and remission rates without declaring such a priori, which in individual trials may raise suspicion of selective reporting.

With only two studies collecting data at later time points, evidence for treatment effect here was generally considered of low quality, with mean differences of score reductions not consistently sparing the null value. The risk ratio for remission appeared preserved at 12 months $(\mathrm{RR}=1.97,95 \%$ CI 1.51 $2.56)$ and this was moderate-quality evidence.

Stratifying the analysis by therapy type highlighted the relative dominance of the CoBalT study in the pooled result, with the mean difference in BDI scores for adjunctive CBT closely reflecting the overall result (m.d. $=-4.56,95 \%$ CI -7.49 to $-1.63)$. The findings for the other modalities based on single small studies seemed imprecise and benefits found were inconsistent: ISTDP reported only with observer-rated HRSD scores indicated favour towards the intervention (m.d. $=-5.84,95 \% \mathrm{CI}$ -11.22 to -0.46 ); group DBT showed a large reduction in mean BDI score but with even wider confidence intervals crossing the null value (m.d. $=-10.79$, 95\% CI -23.83 to 2.25 ); and IPT showed no difference from TAU (m.d. $=0.80,95 \%$ CI -6.70 to 8.30 ). Ideally, a network meta-analysis would have provided a more enriched comparison of the therapy subtypes, but this requires a lot more trials than have thus far been identified.

The all-cause drop-out from treatments showed no significant differences between intervention arms and TAU, with zero heterogeneity, which was therefore considered high-quality evidence of good acceptability. Unfortunately, without data on reasons for study withdrawal, in particular withdrawal due to adverse events, assessment of tolerability was not possible from this review. Two studies did record serious adverse events, and these were observed only in the control arm.

Of the other secondary outcomes, QoL data was the most widely collected (five studies), although all used different scales. No significant differences were found between treatment groups in any study, bar an improvement on the mental subscale of the 12-Item Short Form Health Survey (SF-12) in one study treatment arm. A marginal improvement in social functioning was seen in certain domains in the one study collecting these data but this was not consistent across observer-rated and self-rated scales.

Interestingly, the CoBalT study (Wiles 2016) conducted a cost-utility analysis for CBT, which indicated a cost per quality-adjusted life-year (QALY) gain of $£ 14911$ (ranging from $£ 13006$ to $£ 29626$ based on sensitivity analyses). Based on a societal willingness to pay of $£ 20000$ per QALY, this yielded a high probability (0.92) of cost-effectiveness, which adds weight to its adoption in UK clinical practice.

\section{Discussion}

Overall, this review found moderate-quality evidence that adding psychotherapy to usual care with an antidepressant in treatment-resistant depression was beneficial for depressive symptoms and for response and remission rates over the short term, and high-quality evidence that augmentation had good acceptability. A closer evaluation of these findings is merited to determine any potential implications for clinical practice.

\section{Assessment of reliability (bias)}

As is generally unavoidable for psychotherapy interventions because of lack of masking ('blinding') (Box 2), all included studies were appropriately flagged for high-risk of performance bias and detection bias for the subjective scores, which may overestimate the treatment effect. However, similar improvements rated by masked independent observers on the HRSD suggests this may not be of great concern.

This review might be vulnerable to small-study effects, given the relative dominance of smaller studies addressing the clinical question (three out of the six included here had $<50$ participants). This may be predicted to be of low impact, given that the pooled result favouring psychotherapy is lower than that found in the CoBalT study (Wiles 2016) and one included study reported no benefit (Souza 2016). Ultimately, formal evaluation of publication bias was not possible, because there were insufficient studies for a funnel plot (Box 3), and this would be desirable as more trials are conducted. Three review authors themselves also reasonably declared a conflict of interest, having led the two UK studies of CBT. Although this would not interfere with analysis of included studies, one possible introduction of bias could be the influence of their 
BOX 2 Lack of masking in psychiatric studies

The cornerstone of high-quality studies is to eliminate as far as possible any differences between the intervention and control arms of the study other than the active ingredient of the treatments. Masking ('blinding') to the treatment condition (note that this persists throughout the study, making it distinct from allocation concealment) minimises participants in one group being exposed to different influences compared with another group that may alter the impact of treatment - these influences are broad but could include the nature of contact with healthcare professionals, or reflect a belief about a particular treatment. When such systematic differences between groups occur, it is known as performance bias. If similar different influences are present when outcome data are collected, this is known as detection bias. Failure to mask has been estimated to cause a $23 \%$ exaggeration of intervention effect estimates in trials with subjective outcomes (Balk 2002). Regrettably, there are numerous interventions with potentially vital roles in psychiatry that are practically impossible (or very difficult) to adequately mask, including psychotherapies, physical treatments such as electroconvulsive therapy and, more experimentally, psychoactive drugs such as ketamine and psychedelics that elicit very characteristic reactions. In such instances, although performance bias may be hard to overcome, overall bias of the study (particularly detection bias) can be minimised by including observer-rated outcomes with an independent masked assessor or identifying clearly objective outcomes such as hospital admissions, although these may be less sensitive.

\section{BOX 3 Small-study effects and funnel plots}

It is well described that smaller studies sometimes show different and potentially larger effect sizes for a given intervention than larger studies (Sterne 2000). This is thought to have multiple origins. First, publication bias - i.e. undue influence of the result itself on the decision to publish - may be more likely in smaller studies, as authors may be more inclined to submit smaller studies, and journals themselves more likely to publish them, in instances where results are 'significant'. Other factors include higher possibilities of selective reporting and recruitment of less representative samples. The net effect of cumulative biases from smaller studies may in turn affect the result of meta-analyses.
The best method to test for publication bias among small studies is to visualise through funnel plots whether studies with small/negative effect sizes are missing. On a funnel plot (Fig. 1) the $y$-axis shows standard error, s.e. (which is smaller for larger studies) in reversed scale, and the $x$-axis shows effect size. When there is no publication bias, studies would lie symmetrically around the pooled effect size (the vertical dashed line in Fig 1), 95\% within the triangular region representing the confidence interval around the central estimate. personal experience on the inclusion criteria and hence scope of the review.

The other potential sources of variation in study quality, such as attrition and treatment fidelity, did not seem to greatly alter the effectiveness when removed from the pooled effect size in the subsequent sensitivity analysis.

\section{Evaluating the clinical significance of the findings}

Although this review reports some positive effect of psychological interventions in treatment-resistant depression, the clinical significance of these findings remains uncertain. The authors note that the mean reduction of depressive symptoms on the BDI $(-4.7$, based on the five studies that used this outcome) is indeed of greater magnitude than the minimum clinically important difference (MCID) previously defined by NICE as at least 3 points. However, this threshold has since been under challenge by more recent evidence suggesting that patients themselves may place greater emphasis on larger relative (rather than absolute) changes to
BDI (Button 2015), and clinical significance may be better assessed by addressing changes in specific functional impairments (Saltiel 2015). Although a secondary outcome, the remission and response rates in the intervention arms do suggest that improvements were likely to be clinically significant. Data in this review for benefits on social functioning and quality of life were scarcer and more inconsistent in their findings.

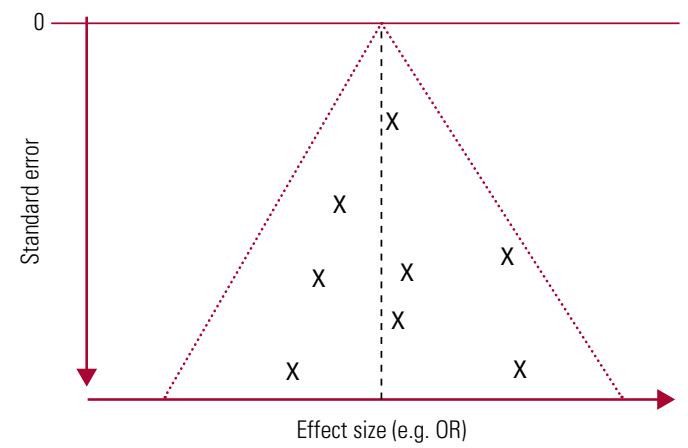

FIG 1 
This review was able to make a confident conclusion about good treatment acceptability based on drop-out rates from the data available but it is becoming increasingly recognised that psychotherapy trials in general often have more limited systems for capturing adverse-event data - either they are not reported or are reported using guidelines developed for drug trials (Duggan 2014). Weaker understanding of tolerability may pose further limitations on the clinical utility of psychotherapy trials, which should readily be addressed in future studies (Linden 2014).

\section{Applicability of the findings}

As previously mentioned, much of the evidence in this review is derived from a single large trial of CBT conducted in primary care. One key consequence for this review is that this clearly weakens the generalisability of the purported pooled result to the other modalities of psychotherapy for which effect sizes were imprecise and less consistent. Of note, however, the severity of symptoms (BDI score of 31.8 in both the intervention and TAU) at baseline in this study was similar to included studies based in secondary care, and therefore whether results could feasibly translate between settings remains an interesting question. Addressing this would ultimately benefit from further studies of psychotherapy in treatment-resistant depression among patients under secondary care in the UK.

The treatment-resistant clinical population should also be considered heterogeneous - perhaps more so when defined by single antidepressant failure - with illness severity, number of treatments used and duration of episode having been laid out as potential variables in treatment resistance in the Maudsley Staging Method (Fekadu 2009). For a more granular understanding of treatment effects, ideally meta-analyses would consider such factors as potential effect modifiers. To its credit, this review did identify a priori initial treatment duration and degree of response for subgroup analyses, but this was not ultimately possible with the small number of studies. One would anticipate in clinical practice that augmentation (either psychological or pharmacological) after a single drug treatment is most likely to be attempted when there has been at least partial response to the primary antidepressant, so this analysis would have added to the review's external validity.

Preference for psychological therapies over pharmacological treatment for psychiatric disorders is suggested to be common in a meta-analysis by McHugh et al (2013), although this could be challenged by the limited uptake of cognitive therapy in $\mathrm{STAR}^{*} \mathrm{D}$ (Wisniewski 2007). Given that treatment preference may ultimately influence outcome (Mergl 2011) as well as uptake in clinical practice, it would have been useful if this review had taken into account baseline characteristics such as educational attainment and prior treatment experiences thought to affect patient preference.

\section{Conclusions and implications for research}

In summary, by applying rigorous inclusion standards of case definition and controlled studies, this review provides the strongest clinical evidence yet for a benefit of psychotherapy given in addition to usual care over usual care alone for treatment-resistant depression.

This review could be regarded as confirming that adjunctive psychotherapy in treatment-resistant depression is very much a credible option among the various next-step choices. There remains, however, no great advancement in guiding the choice for clinicians and patients. NNTB calculated for the included studies here appear favourable if considered against those quoted for antidepressant switch (Papakostas 2008) and antipsychotic augmentation (Nelson 2009) but cannot reliably be extrapolated to compare interventions under differing study conditions. It is noted in the STAR ${ }^{*} \mathrm{D}$ study that CBT augmentation and medication augmentation as next steps were similar in overall outcome but that medication augmentation worked faster (Thase 2007).

This review was unable to fulfil a further aim of evaluating psychotherapy alone as a switch option after first-line treatment failure. However, whether patients with moderate to severe depression would abandon a medication-containing strategy after initial treatment failure may be doubtful, suggesting that this is not such an urgent research question.

Given that the psychological interventions vary in their mechanism of action, more evidence is needed as to the relative effectiveness of different modalities, and interpreting their pooled analysis should clearly be treated with caution. Future research should also address the degree of response to the initial medication and severity of symptoms at the time of augmenting with psychotherapy. In addition, further attention in study designs is needed to other domains relevant to patient outcomes, such as treatment preference, improved reporting of adverse events and long-term remission.

\section{Acknowledgements}

The views expressed here are the views of the author and not necessarily those of the National Health Service. 


\section{Declaration of interest}

None.

An ICMJE form is in the supplementary material, available online at https://doi.org/10.1192/bja. 2020.51

\section{References}

Alvarez-Jimenez M, Wade D, Cotton S, et al (2008) Enhancing treatment fidelity in psychotherapy research: novel approach to measure the components of cognitive behavioural therapy for relapse prevention in first-episode psychosis. Australian and New Zealand Journal of Psychiatry, 42: 1013-20. American Psychiatric Association (2010) Practice Guideline for the Treatment of Patients with Major Depressive Disorder. APA.

Balk EM, Bonis PA, Moskowitz H, et al (2002) Correlation of quality measures with estimates of treatment effect in meta-analyses of randomized controlled trials. JAMA, 287: 2973-82.

Button KS, Kounali D, Thomas L, et al (2015) Minimal clinically important difference on the Beck Depression Inventory-II according to the patient's perspective. Psychological Medicine, 45: 3269-79.

Cleare A, Pariante CM, Young AH, et al (2015) Evidence-based guidelines for treating depressive disorders with antidepressants: a revision of the 2008 British Association for Psychopharmacology guidelines. Journal of Psychopharmacology, 29: 459-525

Cooper C, Katona C, Lyketsos K, et al (2011) A systematic review of treatments for refractory depression in older people. American Journal of Psychiatry, 168: 681-8.

Cuijpers P. Andersson G, Donker T, et al (2011) Psychological treatment of depression: results of a series of meta-analyses. Nordic Journal of Psychiatry, 65: 354-64.

Duggan C, Parry G, McMurran M, et al (2014) The recording of adverse events from psychological treatments in clinical trials: evidence from a review of NIHR-funded trials. Trials, 15: 335

Fava M (2003) Diagnosis and definition of treatment-resistant depression. Biological Psychiatry, 53: 649-59.

Fekadu A, Wooderson S, Donaldson C, et al (2009) A multidimensional tool to quantify treatment resistance in depression: the Maudsley staging method. Journal of Clinical Psychiatry, 70: 177-84.

Feldman G, Harley R, Kerrigan M, et al (2009) Change in emotional processing during a dialectical behavior therapy-based skills group for major depressive disorder. Behaviour Research and Therapy, 47: 316-21.

Guyatt GH, Oxman AD, Kunz R, et al (2008) What is "quality of evidence" and why is it important to clinicians? BMJ, 336: 995-8.

Higgins JPT, Altman DG, Gøtzsche PC, et al (2011) The Cochrane Collaboration's tool for assessing risk of bias in randomised trials. $B M J, 343:$ d5928.

Higgins JPT, Thomas J, Chandler J, et al (2019) Cochrane Handbook for Systematic Reviews of Interventions (2nd edn). Wiley Blackwell.

ljaz S, Davies P, Williams CJ, et al (2018) Psychological therapies for treatment-resistant depression in adults. Cochrane Database of Systematic Reviews, 5: CD010558. Available from: https://doi.org/10. 1002/14651858.CD010558.pub2.

Linden M, Schermuly-Haupt ML (2014) Definition, assessment and rate of psychotherapy side effects. World Psychiatry, 13: 306-9.

McCrone P, Dhanasiri S, Patel A, et al (2008) Paying the Price: The Cost of Mental Health Care in England to 2026. King's Fund.

McHugh RK, Whitton SW, Peckham AD, et al (2013) Patient preference for psychological vs pharmacologic treatment of psychiatric disorders: a meta-analytic review. Journal of Clinical Psychiatry, 74: 595-602.

McIntyre RS, Filteau MJ, Martin L, et al (2014) Treatment-resistant depression: definitions, review of the evidence, and algorithmic approach. Journal of Affective Disorders, 156: 1-7.
McPherson S, Cairns P, Carlyle J, et al (2005) The effectiveness of psychological treatments for treatment-resistant depression: a systematic review. Acta Psychiatrica Scandinavica, 111: 331-40.

Mergl R, Henkel V, Allgaier AK (2011) Are treatment preferences relevant in response to serotonergic antidepressants and cognitive-behavioral therapy in depressed primary care patients? Results from a randomized controlled trial including a patients' choice arm. Psychotherapy and Psychosomatics, 80: 39-47.

Nakagawa A, Mitsuda D, Sado M, et al (2017) Effectiveness of supplementary cognitive-behavioral therapy for pharmacotherapy-resistant depression: a randomized controlled trial. Journal of Clinical Psychiatry, 78: $1126-35$

National Institute for Health and Care Excellence (NICE) (2009) Depression in Adults: Recognition and Management (Clinical Guideline CG90). NICE.

Nelson JC, Papakostas GI (2009) Atypical antipsychotic augmentation in major depressive disorder: a meta-analysis of placebo-controlled randomized trials. American Journal of Psychiatry, 166: 980-91.

Papakostas GI, Fava M, Thase ME (2008) Treatment of SSRI-resistant depression: a meta-analysis comparing within- versus across-class switches. Biological Psychiatry, 63: 699-704.

Ruhé HG, van Rooijen G, Spijker J, et al (2012) Staging methods for treatment resistant depression: a systematic review. Journal of Affective Disorders, 137: 35-45

Saltiel PF, Silvershein DI (2015) Major depressive disorder: mechanismbased prescribing for personalized medicine. Neuropsychiatric Disease and Treatment, 11: 875-88

Shelton RC, Papakostas GI (2008) Augmentation of antidepressants with atypical antipsychotics for treatment-resistant major depressive disorder. Acta Psychiatrica Scandinavica, 117: 253-9.

Souza LH, Salum GA, Mosqueiro BP, et al (2016) Interpersonal psychotherapy as add-on for treatment-resistant depression: a pragmatic randomized controlled trial. Journal of Affective Disorders, 193: 373-80.

Sterne JA, Gavaghan D, Egger M (2000) Publication and related bias in meta-analysis: power of statistical tests and prevalence in the literature. Journal of Clinical Epidemiology, 53: 1119-29.

Stimpson N, Agrawal N, Lewis G (2002) Randomised controlled trials investigating pharmacological and psychological interventions for treatment-refractory depression: systematic review. British Journal of Psychiatry, 181: 284-94.

Thase ME, Friedman ES, Biggs MM, et al (2007) Cognitive therapy versus medication in augmentation and switch strategies as second-step treatments: a STAR*D report. American Journal of Psychiatry, 164: 739-52.

Thomas L, Kessler D, Campbell J, et al (2013) Prevalence of treatmentresistant depression in primary care: cross-sectional data. British Journal of General Practice, 63: e852-8.

Town JM, Abbass A, Stride C, et al (2017) A randomised controlled trial of intensive short-term dynamic psychotherapy for treatment resistant depression: the Halifax Depression Study. Journal of Affective Disorders, 214: 15-25

Trivedi MH, Rush AJ, Wisniewski SR, et al (2006) Evaluation of outcomes with citalopram for depression using measurement-based care in $\mathrm{STAR}^{*} \mathrm{D}$ : implications for clinical practice. American Journal of Psychiatry, 163: $28-40$

Trivedi RB, Nieuwsma JA, Williams Jr JW (2011) Examination of the utility of psychotherapy for patients with treatment resistant depression: a systematic review. Journal of General Internal Medicine, 26: 643-50.

Wiles NJ, Hollinghurst S, Mason V, et al (2008) A randomized controlled trial of cognitive behavioural therapy as an adjunct to pharmacotherapy in primary care based patients with treatment resistant depression: a pilot study. Behavioural and Cognitive Psychotherapy, 36: 21-33.

Wiles NJ, Thomas L, Turner N, et al (2016) Long-term effectiveness and cost-effectiveness of cognitive behavioural therapy as an adjunct to pharmacotherapy for treatment-resistant depression in primary care: follow-up of the CoBalT randomised controlled trial. Lancet Psychiatry, 3: 137-44.

Wisniewski SR, Fava M, Trivedi MH, et al (2007) Acceptability of secondstep treatments to depressed outpatients: a STAR*D report. American Journal of Psychiatry, 164: 753-60. 